\title{
Elongation of confined ferrofluid droplets under applied fields
}

\author{
S. Banerjee ${ }^{1}$, M. Fasnacht ${ }^{1}$, S. Garoff ${ }^{1,2}$ and M. Widom ${ }^{1}$ \\ ${ }^{1}$ Department of Physics \\ ${ }^{2}$ Colloids, Polymers, and Surfaces Program \\ Carnegie Mellon University, Pittsburgh, Pa. 15213
}

(August 8, 2018)

\begin{abstract}
Ferrofluids are strongly paramagnetic liquids. We study the behavior of ferrofluid droplets confined between two parallel plates with a weak applied field parallel to the plates. The droplets elongate under the applied field to reduce their demagnetizing energy and reach an equilibrium shape where the magnetic forces balance against the surface tension. This elongation varies logarithmically with aspect ratio of droplet thickness to its original radius, in contrast to the behavior of unconfined droplets. Experimental studies of a ferrofluid/water/surfactant emulsion confirm this prediction.
\end{abstract}

\section{INTRODUCTION}

Ferrofluids [1] are oil- or water-based colloidal suspensions of permanently magnetized particles. In an applied magnetic field the particles align creating a strong paramagnetic response in the ferrofluid. Because they are fluids, these suspensions can flow in response to forces. For example, ferrofluid droplets elongate parallel to applied fields [2 5, 7,6] and undergo tip-sharpening transitions [8,9. When a ferrofluid droplet is confined between two plates in a "thin film" geometry, surrounded by an immiscible fluid, and a field is applied perpendicular to the plates, it undergoes field induced bifurcations [10] leading to intricate labyrinthine patterns [11]. Ferrofluid emulsions [12] undergo structural transitions under an applied field from a randomly dispersed structure of the emulsion droplets to droplet chains, columns and worm like structures [13,14 depending on volume fraction, sample geometry and the rate of field application.

A droplet of ferrofluid elongates under applied field because of the demagnetizing fields of magnetic poles on the surface of the droplet. Surface poles arise wherever the droplet magnetization has a component perpendicular to the surface. The demagnetizing field that they create opposes the magnetization, creating a demagnetizing energy that depends on the shape of the droplet. The droplet elongates to reduce its demagnetizing field and energy. Because elongation increases the surface energy of the system, an equilibrium shape is reached when the magnetic forces balance against the surface tension forces.

The elongation of freely suspended, 3-dimensional droplets has been well studied [2 [5]. The droplets can be assumed to be ellipsoids for small elongation. The demagnetizing field is thus uniform and the elongation (major axis minus minor axis divided by minor axis) is found to be proportional to the undeformed droplet radius. The case of droplets confined in "thin film geometry" however, involves two length scales, droplet thickness and its undeformed diameter. In the limit of small aspect ratio (droplet thickness divided by its undeformed diameter) the demagnetizing fields are stronger near the edges of the droplet than at its center. We find that the elongation divided by droplet thickness in this geometry is proportional to the logarithm of the aspect ratio.

Prior experiments [6,7] have proposed droplet elongation as a tool for measuring surface tension between the ferrofluid and the surrounding immiscible fluid. We improve on the existing theory [6] by incorporating spatial variation of the demagnetizing field inside the droplet. We perform an experiment supporting our predicted logarithmic behavior.

Section II of this paper presents our theoretical study of the elongation of a ferrofluid droplet confined within a thin film. Our principal result is a predicted logarithmic dependence of elongation on droplet aspect ratio. We contrast this result with the corresponding elongation of unconfined droplets. Section III describes an experiment done with ferrofluid emulsions that tests our theory. The experiment is in qualitative agreement with our theoretical prediction, but differs quantitatively in at least one respect. In section IV we discuss a possible explanation of the discrepancy based upon droplet contact angles with the confining plates. 


\section{THEORY}

Consider a paramagnetic liquid droplet confined in a thin film between two parallel plates with a gap, $\Delta$, in the $\hat{z}$ direction (see figure 1). An immiscible liquid surrounds the droplet. Let the thickness, $\Delta$, be much smaller than the radius of the undeformed droplet, $r_{0}$. This small aspect ratio

$$
p=\frac{\Delta}{2 r_{0}}
$$

provides the pseudo-two-dimensional character of the problem. If a uniform, weak, field $\mathbf{H}_{0}$ is applied parallel to the plate, the droplet magnetizes. The magnetization creates an opposing demagnetizing field whose strength depends on the droplet shape. The droplet elongates to decrease its magnetic energy, reaching equilibrium when the magnetic forces balance against the restoring forces due to surface tension. In this section we define the elongation of the droplet and calculate the surface energy, $E_{S}$, and the magnetic energy, $E_{M}$, of the droplet as a function of its elongation. By minimizing the total energy with respect to the elongation we obtain the elongation as a function of $\mathbf{H}_{0}, r_{0}$, and $\Delta$.

For simplicity assume the elongated droplet has a uniform cross section, $\mathcal{C}$, independent of $z$. This corresponds to a contact angle of $90^{\circ}$ between the paramagnetic liquid, the surrounding fluid and the glass plates, and a plate spacing much less than the capillary length of the two liquids. Thus the droplet has straight edges if viewed from the side (see figure 1). The role of contact angle will be discussed later in section IV. We write the equation for $\mathcal{C}$ in polar coordinates as a generic smooth perturbation to a circle,

$$
r=\alpha_{1}+\alpha_{2} \cos 2 \theta .
$$

We only include a single harmonic, since we expect coefficients for the higher harmonics to be much smaller than $\alpha_{2}$ for small perturbations. The cross section $\mathcal{C}$ has semi-major axis $a$, and semi-minor axis $b$ (see figure $1 \mathrm{~b}$ ), with $\alpha_{1}=(a+b) / 2$ and $\alpha_{2}=(a-b) / 2$. We define the elongation of the droplet

$$
\epsilon \equiv \frac{a}{b}-1
$$

We assume that the elongation, $\epsilon$, is much less than 1 . Imposing the constraint that the volume of the droplet $(\Delta$ times cross-sectional area) remains constant we calculate

$$
\alpha_{1}=\frac{r_{0}}{\left(1+k^{2} / 2\right)^{1 / 2}}, \quad \alpha_{2}=\frac{r_{0} k}{\left(1+k^{2} / 2\right)^{1 / 2}}
$$

where $k=\epsilon /(2+\epsilon)$.

The surface energy is the sum of interfacial areas times surface tensions between all pairs of the three phases (solid glass, ferrofluid droplet and immiscible fluid). For the case of uniform cross-section ( $90^{\circ}$ contact angle) droplets, the glass-ferrofluid and glass-immiscible fluid interfacial areas are independent of the shape of $\mathcal{C}$ due to the fixed volume constraint. Hence we concern ourselves with the droplet-surfactant solution interface, the area of which is $\Delta$ times the perimeter. The perimeter of cross section $\mathcal{C}$ can be calculated as a power series in $\epsilon$,

$$
S=2 \pi r_{0}\left(1+\frac{3}{16} \epsilon^{2}+O\left(\epsilon^{3}\right)\right) .
$$

As expected, the leading correction to $S$ is second order in $\epsilon$ since the perimeter should increase regardless of the sign of $\epsilon$. The relevant surface energy of the droplet is

$$
E_{S}=\sigma_{F I} S \Delta
$$

where $\sigma_{F I}$ is the surface tension of the ferrofluid-immiscible fluid interface.

The total magnetic energy of any paramagnetic body under applied field is [15]

$$
E_{M}=-\frac{1}{2} \int_{V} d^{3} \mathbf{r} \mathbf{H}_{0} \cdot \mathbf{M}(\mathbf{r}) .
$$

The magnetization $\mathbf{M}(\mathbf{r})$ is determined by the self consistent equation

$$
\mathbf{M}(\mathbf{r})=\chi\left(\mathbf{H}_{0}+\mathbf{H}_{D}(\mathbf{r})\right)
$$


for linear susceptibility $\chi$, where

$$
\mathbf{H}_{D}(\mathbf{r})=\int_{S} d^{2} \mathbf{r}^{\prime}\left(\mathbf{M}\left(\mathbf{r}^{\prime}\right) \cdot \hat{\mathbf{n}}\left(\mathbf{r}^{\prime}\right)\right) \frac{\mathbf{r}-\mathbf{r}^{\prime}}{\left|\mathbf{r}-\mathbf{r}^{\prime}\right|^{3}}+\int_{V} d^{3} \mathbf{r}^{\prime}\left(\nabla \cdot \mathbf{M}\left(\mathbf{r}^{\prime}\right)\right) \frac{\mathbf{r}-\mathbf{r}^{\prime}}{\left|\mathbf{r}-\mathbf{r}^{\prime}\right|^{3}}
$$

is the demagnetizing field due to the magnetization $\mathbf{M}(\mathbf{r})$, with $\hat{\mathbf{n}}\left(\mathbf{r}^{\prime}\right)$ being the outward normal at any point on the surface. The surface integral gives the demagnetizing field due to the surface poles which appear wherever the magnetization has a component normal to the surface. The volume integral gives the contribution to the demagnetizing field due to volume charges which appear at points where the magnetization has non-zero divergence.

To calculate the magnetic energy we expand $\mathbf{M}$ and $\mathbf{H}_{D}$ in power series in the susceptibility $\chi$,

$$
\begin{aligned}
& \mathbf{M}(\mathbf{r})=\mathbf{M}^{(1)}(\mathbf{r})+\mathbf{M}^{(2)}(\mathbf{r})+\mathbf{M}^{(3)}(\mathbf{r})+\ldots \\
& \mathbf{H}_{D}(\mathbf{r})=\mathbf{H}_{D}^{(1)}(\mathbf{r})+\mathbf{H}_{D}^{(2)}(\mathbf{r})+\mathbf{H}_{D}^{(3)}(\mathbf{r})+\ldots
\end{aligned}
$$

where $\mathbf{M}^{(n)}(\mathbf{r})$ and $\mathbf{H}_{D}^{(n)}(\mathbf{r})$ are proportional to $\chi^{n}$. Equating terms in (8) of equal order in $\chi$ we get

$$
\mathbf{M}^{(1)}(\mathbf{r})=\chi \mathbf{H}_{0}
$$

and

$$
\mathbf{M}^{(n+1)}(\mathbf{r})=\chi \mathbf{H}_{D}^{(n)}(\mathbf{r}) .
$$

Note that $\mathbf{M}^{(1)}(\mathbf{r})$ is independent of $\mathbf{r}$ because the applied field is uniform whereas $\mathbf{M}^{(n)}(\mathbf{r})$ may depend on $(\mathbf{r})$ for $n>1$ because $\mathbf{H}_{D}(\mathbf{r})$ may be non-uniform. To second order in $\chi$ we write the magnetic energy of the droplet in (7) as

$$
E_{M}=-\frac{1}{2} \chi H_{0}^{2} V-\frac{1}{2} \int_{V} d^{3} \mathbf{r} \mathbf{M}^{(1)} \cdot \mathbf{H}_{D}^{(1)} .
$$

The first term in equation (14) for the magnetic energy is independent of the shape of the droplet and hence unimportant for our consideration. The second term in the energy is the demagnetizing energy $E_{D}$ due to a uniform magnetization $\mathbf{M}^{(1)}=\chi \mathbf{H}_{0}$. Because $\mathbf{M}^{(1)}$ is uniform there are no volume charges, and the surface poles appear only along the droplet-immiscible fluid interface, to first order in $\chi$. Rewrite the second term in (14) as an energy due to the induced surface charges along the curved surface of the droplet

$$
E_{D}=\frac{1}{2} \chi^{2} \int_{0}^{\Delta} d z \int_{0}^{\Delta} d z^{\prime} \oint d s \oint d s^{\prime} \frac{\left(\hat{\mathbf{n}} \cdot \mathbf{H}_{0}\right)\left(\hat{\mathbf{n}}^{\prime} \cdot \mathbf{H}_{0}\right)}{\left|\mathbf{r}-\mathbf{r}^{\prime}\right|} .
$$

Here $d s$ and $d s^{\prime}$ are infinitesimal arc-lengths along the contour of the droplet $\mathcal{C}$, and $\hat{\mathbf{n}}$ and $\hat{\mathbf{n}}^{\prime}$ are the outward normals to the curved surface of the droplet at points $(s, z)$ and $\left(s^{\prime}, z^{\prime}\right)$ respectively.

Write $\left|\mathbf{r}-\mathbf{r}^{\prime}\right|=\sqrt{R^{2}+\left(z-z^{\prime}\right)^{2}}$, where $R$ is the in-plane distance between points at positions $s$ and $s^{\prime}$ on $\mathcal{C}$. Integrating over $z$ and $z^{\prime}$ in (15) yields [10]

$$
E_{D}=\chi^{2} \Delta \oint d s \oint d s^{\prime}\left(\hat{\mathbf{n}} \cdot \mathbf{H}_{0}\right)\left(\hat{\mathbf{n}}^{\prime} \cdot \mathbf{H}_{0}\right) \Phi(R / \Delta)
$$

where

$$
\Phi(R / \Delta)=R / \Delta-\sqrt{1+(R / \Delta)^{2}}+\ln \left[(R / \Delta) /\left(\sqrt{1+(R / \Delta)^{2}}-1\right)\right] .
$$

Using equation (2) for $\mathcal{C}$ we calculate the demagnetizing energy in (16) as a series expansion in $\epsilon$ and the aspect ratio $p=\Delta / 2 r_{0}$

$$
E_{D}=\chi^{2} H_{0}^{2} V\left\{2 p \ln \frac{B}{p}-3 \epsilon p \ln \frac{C}{p}+\cdots\right\}
$$

where $V=\pi r_{0}^{2} \Delta$ is the volume of the droplet, and $B=4 e^{-1 / 2}$ and $C=4 e^{-5 / 6}$ are geometrical constants. The term in the brackets can be identified as $2 \pi$ times the demagnetizing factor [15] of the droplet along the direction of applied 
field. Additional terms in the series in equation (18) are of higher order in $\epsilon$ or in $p$. For small elongation and large aspect ratio we may neglect these higher order terms.

Minimizing the total energy $E=E_{S}+E_{M}$ with respect to $\epsilon$ gives

$$
\epsilon=\frac{\chi^{2} H_{0}^{2} \Delta}{\sigma_{F I}} \ln \frac{C}{p} .
$$

Corrections to this result are higher order in aspect ratio $p$ or higher order in $\epsilon$ itself. Interestingly, the elongation depends only logarithmically on the undeformed radius $r_{0}$, and has a much stronger dependence on the thickness, $\Delta$, of the droplet. This result differs from an earlier theory [6] which omits the logarithm because it assumes that the demagnetizing field is uniform inside the droplet.

In the case of unconfined, nearly ellipsoidal droplets [4,5], the demagnetizing field is quite uniform inside the droplet. The demagnetizing energy is therefore proportional to the volume $\left((4 / 3) \pi r_{0}^{3}\right)$ of the droplet according to equation (14). The surface energy is proportional to the area $\left(4 \pi r_{0}^{2}\right)$ and the elongation is thus proportional to $r_{0}$. In the case of thin film geometry, however, the demagnetizing field is very non-uniform. For distances much less than $\Delta$ near the droplet edge, the component of the demagnetizing field is of order $M$, since the edge acts like an infinite sheet of charge in the first approximation. For distances much greater than $\Delta$ the demagnetizing field is of order $M \Delta / r$ since the edge acts as a line charge in this case. The contribution to the integral for the demagnetizing energy in equation (14) mainly comes from the bulk of the droplet and goes like $r_{0} \Delta^{2} \ln \left(r_{0} / \Delta\right)$. The surface energy is proportional to $2 \pi r_{0} \Delta$ and the elongation is therefore proportional to $\Delta \ln \left(r_{0} / \Delta\right)$. The logarithmic variation of elongation with the aspect ratio is thus a signature of the non-uniform nature of the demagnetizing field inside the droplet.

\section{EXPERIMENT}

\section{A. Setup}

a. Sample Preparation and Structure Our sample consisted of a ferrofluid/aqueous solution emulsion confined between two glass plates. The oil-based ferrofluid used was EMG 905 made by Ferrofluidics. To reduce the surface tension between the ferrofluid and the immiscible aqueous external phase, we incorporated surfactants in the aqueous phase. A solution of a commercial detergent made the best emulsions while solutions with other pure anionic surfactants either showed hardly any elongation of the ferrofluid droplets under applied field or produced droplets without sharp boundaries with the aqueous phase. In contrast, our stable, well behaved emulsions allowed us to probe and confirm the fundamental aspects of our model.

To prepare the emulsions, a single drop of ferrofluid $(\sim 0.1 \mathrm{ml})$ was added to $10 \mathrm{ml}$ of surfactant solution which was a 12 times dilution of the commercial detergent. The liquid was shaken (by hand) to prepare the emulsion, creating ferrofluid droplets with diameters varying from $\sim 5-200 \mu \mathrm{m}$. A small amount of this emulsion was then put between two glass plates which were circular, about $2 \mathrm{~cm}$ in diameter and $4 \mathrm{~mm}$ in thickness. These plates were cleaned using soap and alcohol and then rinsed with ROPure water. We also tried acid cleaning of the glass plates, however it did not result in any noticeable change in the quality of the sample.

We used a rectangular spacer made of mylar foil to separate the plates and prevent the emulsion from leaking out from the edges of the plates. The mylar foil extended to to the edges of the glass plates and had a rectangular hole in the center into which the emulsion was inserted. The thickness of a single mylar spacer was measured to be $6.54 \pm 0.06 \mu \mathrm{m}$. The experiment was performed with one and two spacers to ensure small aspect ratio.

For the cell assembly, the mylar spacers were placed on the first plate and a drop of the emulsion was put in the center of the plate. The second plate was placed on top and the two plates were clamped together using a pair of brass rings. The rings were tightened by a set of 4 equally spaced screws. We measured the thickness variation across the sample by making a "dry" sample (without the emulsion) and counting resulting white light interference fringes. Although the thickness of mylar spacers was measured to an accuracy of 1 percent, the thickness variation across the sample was found to be $10 \%$ resulting from the stresses due to clamping and possible entrapment of dust in the cell.

b. Apparatus A schematic diagram of the experimental setup is shown in figure 2 . We put the sample at the center of a pair of Helmholtz coils to insure a homogeneous magnetic field. The field measured close to the sample using a Hall probe showed a variation of less than $4 \%$ across the sample. The sample was set up horizontally to prevent gravitational settling of the ferrofluid droplets. Horizontal alignment was achieved using a bubble level.

The sample was illuminated from below using a diffused light source and observed from above using a telemicroscope. The tele-microscope was connected to a CCD camera and the image from it was fed into a video recorder and recorded on video tape. Images from the recording were later processed using NIH Image. We calibrated the optical system using a measuring reticule aligned along the two orthogonal directions of the CCD array. Figure 
3 shows a low magnification view of a typical sample. The ferrofluid droplets appear much darker in the image than the surfactant solution around them.

c. Experimental Procedure and Image Analysis During the experiment the applied field was incremented every few seconds. We found the response of the droplets to the field to be nearly instantaneous and the shape of the droplets remained constant at constant field. Experiments with decreasing field strength showed no hysteresis in droplet shape. While droplet elongations were observed to be small we incremented the field in steps of about 1 Gauss, and increased the increments up to about 5 Gauss as the elongation increased. Droplet elongations appeared to vary smoothly with applied fields over the entire range from 0 to 50 Gauss.

During each experiment the droplets were observed on a video monitor and recorded on tape. After grabbing images of distorted droplets, we used a cut-off in pixel gray scale level to identify the droplet edge. The semi-major axis $(a)$ and the semi-minor axis $(b)$ were directly read off the image using NIH Image. At zero field measured elongations were small (RMS magnitude around 0.003) and in random directions. These minor perturbations from a circular shape were likely due to microscopic distortion of the contact line pinned on weak surface heterogeneities. The "observed radius" $r_{0}$ was calculated as the average of the two semi-axes at zero field and the elongation at each field value was calculated using data analysis software.

d. Results For each of the 48 droplets studied we plotted elongation $\epsilon$, versus the square of the applied field, $\mathbf{H}_{0}$. Figure 4 shows typical plots. The elongation is proportional to the square of the applied field for small applied fields as predicted. Saturation effects, although small, can be seen at higher values of the field. The plot of elongation, for each droplet was fitted to

$$
\frac{\epsilon}{\Delta}=k_{0}+k_{1} H_{0}^{2}+k_{2} H_{0}^{4}
$$

We included terms only up to order $H_{0}^{4}$ because the saturation effects were observed to be small. We include $k_{0}$ to allow for the observed small elongations at zero field.

The coefficients $k_{1}$ of each droplet were then plotted versus the inverse of the aspect ratio $1 / p=2 r_{0} / \Delta$ on a semi-log plot (see figure 5). The theory predicts a slope of $\chi^{2} / \sigma_{F I}$ and an intercept of $1 / C$ on the horizontal axis with $C=1.74$. The data points in figure 5(a) fall on a straight line as predicted by the theory. Also, as predicted by the theory, the data points for two different droplet thicknesses overlay each other. There is substantial scatter in the data, but the deviations from a straight line are random and consistent with the error bars. The chief source of uncertainty was the $10 \%$ uncertainty in thickness due to the variation observed across the sample. Figure 5(b) displays the deviation of $k_{1}$ from the best fit normalized by the uncertainty. The uncertainties in measuring $\epsilon, r_{0}$ and $\mathbf{H}_{0}$ were found to be negligible in comparison.

Dividing the susceptibility $\chi=1.9$ for the ferrofluid used [16] by the slope $=0.119 \pm 0.004 \mathrm{~cm} /$ dyne obtained from the fitted line we get $\sigma_{F I}=30.4 \pm 1.1$ dynes $/ \mathrm{cm}$, typical of oil-water surface tensions. From the fitted line we also get $C=0.35 \pm 0.08$, differing substantially from our theoretically predicted value of 1.74 . It may be possible to explain this discrepancy by considering the effect of the contact angle of the ferrofluid-immiscible fluid interface with the glass plates. In the discussion section below we explore the qualitative effect of the contact angle.

In figure 6(a) we plot $\epsilon / \Delta$ versus $2 r_{0} / \Delta$ on a linear scale. If the demagnetizing field inside the droplet was uniform like in the case of unconfined droplets, the plot would be a straight line. However, the plot is clearly not a straight line and the deviations from the best fitted straight line are systematic (see figure 6(b)). This further supports our theoretical result that the demagnetizing field inside a confined droplet is non-uniform and the elongation divided by thickness is proportional to the logarithm of the aspect ratio.

\section{DISCUSSION}

The results discussed in section III agree with our theoretical prediction (19) of logarithmic variation of $\epsilon / \Delta$ with a proportionality constant of $\chi^{2} / \sigma_{F I}$. However, our theoretical value for $C$ is $4 e^{-5 / 6}=1.74$ whereas the experimentally measured value for $C$ is $0.35 \pm 0.08$.

One possible explanation for the discrepancy in the value of $C$ is that the ferrofluid/glass contact angle is not $90^{\circ}$ and consequently the cross-section of the droplet is not uniform. Our calculations are for uniform droplet cross-section, which corresponds to a contact angle $\beta=90^{\circ}$ between the glass plate and liquid droplet. The experiment, however, was performed with an oil-based ferrofluid in a surfactant solution for which the oil-glass contact angle $\beta<90^{\circ}$ (see figure 7). A contact angle of other than $90^{\circ}$ will affect the elongation in two ways: by changing interfacial areas to alter the functional form of $E_{S}$ and by redistributing the magnetic surface poles to alter the functional form of $E_{M}$. We consider these two effects in turn. First, however, we must address an ambiguity in the definition of aspect ratio and elongation which results from the non-uniformity of droplet cross-section. 
Our experiment observes the profile of the largest cross-section of the droplet. For a circular droplet with $\beta<90^{\circ}$ this is the radius $r_{1}$ defined as the radius at mid-gap as shown in figure 7. For an elongated droplet we measure the semi-major and -minor axes $a_{1}$ and $b_{1}$ and, through equation (3), the elongation $\epsilon_{1}$. We also define $r_{2}, a_{2}, b_{2}$ and $\epsilon_{2}$ associated with the ferrofluid-immiscible fluid-glass plate contact line (see figure 7 ). Since $\Delta$ is much less than the capillary length of the ferrofluid/immiscible fluid, to a good approximation 17] the profile of the droplet will be an arc of a circle, so the difference between $r_{1}$ and $r_{2}$ is of order $\Delta$, and likewise for the semi-major and -minor axes. The difference $\epsilon_{1}-\epsilon_{2}$ is of order $\Delta / r_{1}$ relative to the elongation. Recall that our result (19) for the elongation is only the lowest order term in a series expansion in the aspect ratio. Thus the distinction between $r_{1}$ and $r_{2}$, and between $\epsilon_{1}$ and $\epsilon_{2}$, does not alter our result at the lowest order in aspect ratio.

When the contact angle differs from $90^{\circ}$, the cross section of the droplet depends on $z$. Consequently, the contact areas of the glass plates with the droplet and with the surfactant solution may vary as the droplet elongates. All the three interfacial areas must be taken into account to calculate the surface energy. The total surface energy is

$$
E_{S}=\sigma_{F I} A_{\mathcal{C}}+2 \sigma_{F G} A_{G}+2 \sigma_{I G}\left(A-A_{G}\right)
$$

where the three surface tensions between ferrofluid-immiscible fluid, ferrofluid-glass, and surfactant solution-glass, are denoted by $\sigma_{F I}, \sigma_{F G}$, and $\sigma_{I G}$ respectively, $A_{C}$ and $A_{G}$ are defined below and the total area of the sample is denoted by $A$. The factors of 2 in the second and third terms of the surface energy account for the two glass surfaces.

The area of the droplet-surfactant solution interface $A_{\mathcal{C}}$ is given approximately by the circumference of $\mathcal{C}$ multiplied by the arc length of the bulge

$$
A_{C}=2 \pi r_{1}\left(1+\frac{3}{16} \epsilon^{2}\right) \Delta \frac{(\pi / 2-\beta)}{\cos \beta} .
$$

We use $r_{1}$ here to calculate the circumference of the droplet because is the radius observed during the experiment. To first order in the aspect ratio, using $r_{1}$ or $r_{2}$ in equation (22) yields the same result.

The droplet's contact area with the glass plates must be adjusted to maintain a constant total volume of ferrofluid as the droplet elongates. We approximate the volume of the bulging region by the circumference of $\mathcal{C}$ multiplied by the projected area of the bulge. The contact area $A_{G}$ must be adjusted so that $A_{G} \Delta$ changes by the negative of the change in volume of the bulge. Thus we write

$$
A_{G}=2 \pi r_{2}^{2}\left[1-\frac{3}{32}\left\{\frac{(\pi / 2-\beta)}{\cos ^{2} \beta}-\tan \beta\right\} \frac{\Delta}{r_{2}} \epsilon^{2}\right] .
$$

Using $r_{2}$ instead of $r_{1}$ makes the above result exact for zero elongation. The area of ferrofluid in contact with the glass plates decreases with elongation for an acute contact angle because the volume of the fluid contained in the outward bulge of the droplet increases and therefore the fluid contained in the bulk of the droplet decreases. For obtuse contact angles exactly the opposite happens for similar reasons.

To understand how the contact angle affects the magnetic energy, consider the work done by the magnetic field as we change the contact angle from $90^{\circ}$ to $\beta$ while keeping the volume of the droplet constant. This work, divided by the circumference, must be independent of $r_{1}$ in the limit $r_{1}$ going to infinity, since the magnetic field near the surface of the droplet will not depend on $r_{1}$ in the large $r_{1}$ limit. The work done by the magnetic field is the difference in energy between the straight edge droplet with contact angle of $90^{\circ}$ and the bulging droplet with a contact angle of $\beta$. The demagnetizing energy of the bulging droplet must therefore have the same dependence on $\ln \left(r_{1} / \Delta\right)$ as the straight edge droplet or the difference in the demagnetizing energies divided by the circumference will be proportional to $\ln \left(r_{1} / \Delta\right)$ and will blow up in the large $r_{1}$ limit. Hence, the demagnetizing energy for the bulging droplet must be identical to equation (18) but with different values $\tilde{B}$ and $\tilde{C}$ replacing the constants $B$ and $C$.

As the droplet bulges inward or outward the charges on the surface get distributed over a larger area, decreasing the demagnetizing energy. The constant $\tilde{B}$ therefore has a smaller value for a bulging (inward or outward) droplet than $B$, the value for a straight-edged droplet. However, since the demagnetizing energy is always positive, smaller demagnetizing energy $(\tilde{B}<B)$ implies a weaker dependence of demagnetizing energy on elongation. Thus, we expect the value of $\tilde{C}$ to be smaller for a bulging droplet than the value $C$ for a straight-edged droplet.

Finally, consider how the contact angle dependence of surface and magnetic energies affect the elongation calculated in equation (19) for the case $\beta=90^{\circ}$. The $\epsilon$ dependence of the surface energy remains quadratic, but the coefficient now depends upon a linear combination of the three surface tensions $\sigma_{F I}, \sigma_{F G}$ and $\sigma_{I G}$. This combination will replace $\sigma_{F I}$ in equation (19). The functional form of the magnetic energy remains unchanged, but the values of $B$ and $C$ depend on contact angle. Thus the smaller value $\tilde{C}$ replaces $C$ in equation (19). For $\beta \neq 90^{\circ}$ the experiment cannot be used to determine $\sigma_{F S}$ unless $\sigma_{F G}$ and $\sigma_{I G}$ are known. Since the $\beta$ in general is not $90^{\circ}$, it is only possible to measure the effective surface tension during elongation, and not $\sigma_{F I}$ itself. 


\section{CONCLUSIONS}

We study the elongation ferrofluid droplets, confined in thin film geometry, under weak applied field. Our theoretical calculations predict the elongation of a droplet depends logarithmically on aspect ratio. This behavior contrasts with the case of unconfined 3-dimensional droplets where elongation is directly proportional to undeformed droplet radius. We measured the elongation of ferrofluid droplets in an experiment performed on ferrofluid droplets in a ferrofluid/water/surfactant emulsion. The results of our experiment agree with the functional form our theoretical

prediction, however the experimentally measured value of $C$ differs from the predicted value. We suggest the droplet contact angle with the confining plates as a source of this discrepancy.

\section{ACKNOWLEDGMENTS}

We acknowledge partial support for this research under NSF grant DMR-9732567.

[1] R.E. Rosensweig, Ferrohydrodynamics (Cambridge, 1985).

[2] V.I. Arkhipenko, Y.D. Barkov, V.G. Bashtovoi, Magnetohydrodynamics 14, 373 (1978).

[3] V.I. Drozdova, T.V. Skrobotova, V.V. Chekanov, Magnetohydrodynamics 15, 16 (1979).

[4] J. C. Bacri and D. Salin, J. Physique. Lett. 43, L-649 (1982).

[5] V.G. Bashtovoi, S.G. Pogirinitsakaya and A.G. Reks, Magnetohydrodynamics 23, 23 (1987).

[6] C. Flament, et al. Phys. Rev. E 53, 4801 (1996).

[7] V.G. Bashtovoi, et al. (unpublished, cited in ref. 6).

[8] J. C. Bacri and D. Salin, J. Physique. Lett. 44, 415 (1983).

[9] H. Li, T.C. Halsey and A. Lobkovsky, Europhys. Lett. 27, 575 (1994).

[10] S.A Langer, R.E. Goldstein and D.P. Jackson, Phys. Rev. A 46, 4894 (1992).

[11] R.E. Rosensweig, M. Zahn and R. Shumovich, J. Magn. Magn. Mater. 39, 127-32 (1983).

[12] J. Bibette, D. Roux and F. Nallet, Phys. Rev. Lett. 65, 2470-3 (1990).

[13] J. Liu, et al. Phys. Rev. Lett. 74, 2828-31 (1995).

[14] G.A. Flores, et al., Int. J. Mod. Phys. B. 10, 3283-92 (1996).

[15] W.F. Brown, "Magnetostatic Principles in Ferromagnetism" (North-Holland, Amsterdam, 1962).

[16] Private communication, Ferrofluidics Inc. (1998).

[17] A.W. Adamson, "Physical Chemistry of Surfaces" (Interscience, 1967). 
$\longrightarrow \mathbf{H}_{0}$

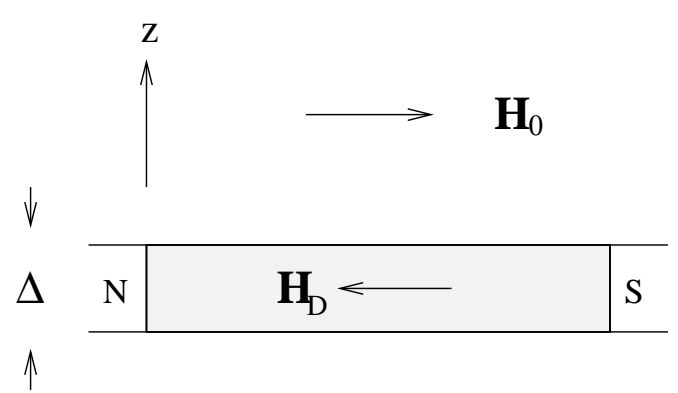

(a)

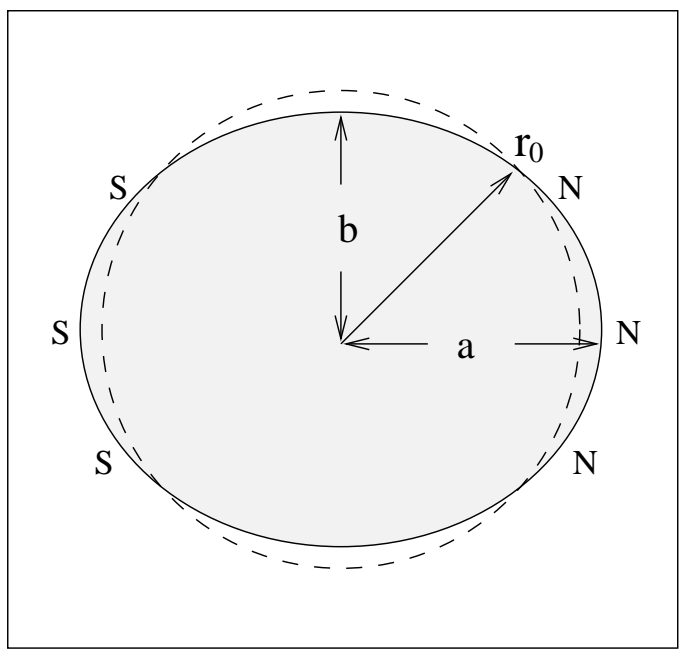

(b)

FIG. 1. (a) A side view of a ferrofluid droplet confined between two glass plates. (b) A top view of a ferrofluid droplet elongating under applied field. The dashed line shows the undeformed droplet. $\mathrm{N}$ and $\mathrm{S}$ indicate the north and south magnetic poles. $\mathbf{H}_{D}$ is the demagnetizing field.

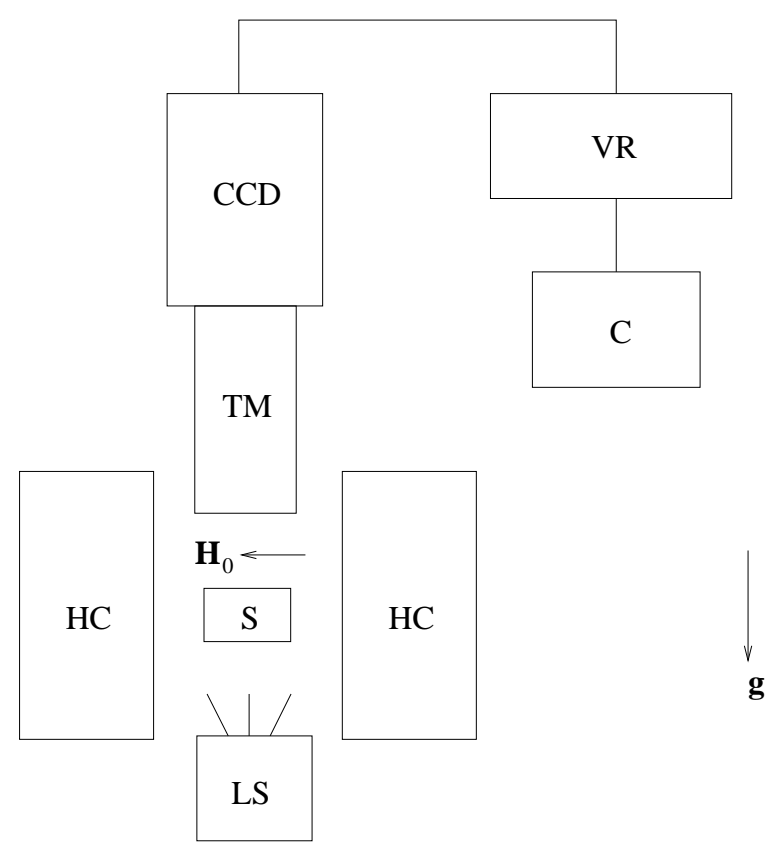

FIG. 2. A schematic diagram of the experimental setup. LS=Light Source, HC=Helmholtz Coil, S=Sample, $\mathrm{TM}=$ Tele-Microscope, $\mathrm{CCD}=\mathrm{CCD}$ Camera, $\mathrm{VR}=$ Video Recorder, $\mathrm{C}=$ Computer 


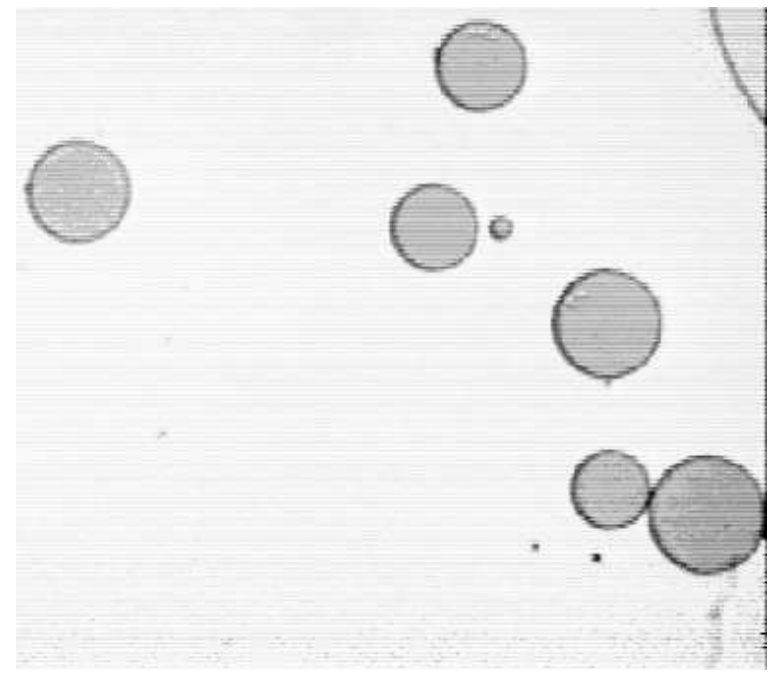

FIG. 3. A small magnification view of the sample showing ferrofluid droplets in emulsion.

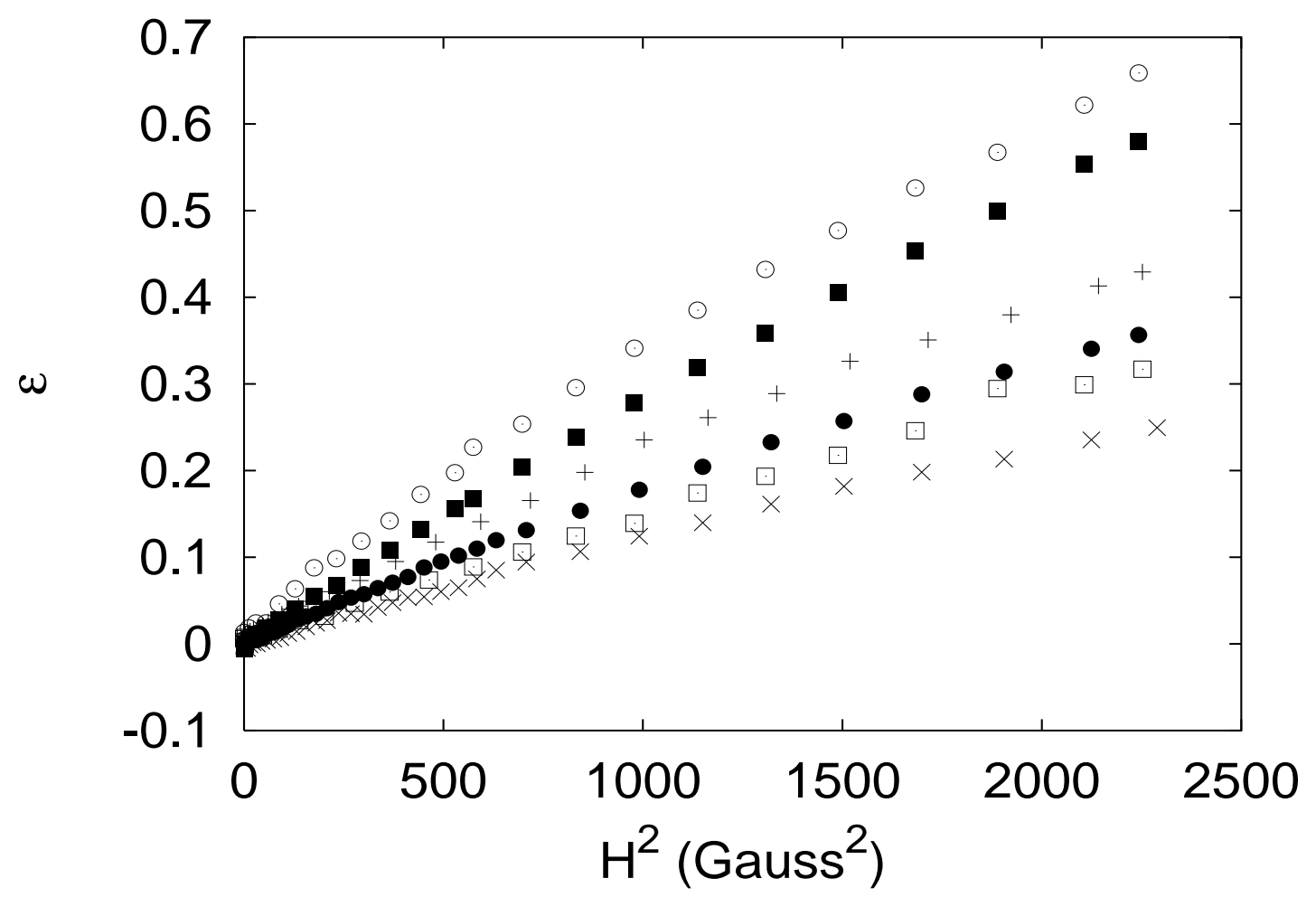

FIG. 4. The plot of elongation vs $H^{2}$ for droplets with different radii and two different thicknesses. The error bars are smaller than the size of the symbols on the plot. The symbols and radii for droplets with thickness spacing of $\Delta=6.5 \mu m$ are $(\times, 96.5 \mu \mathrm{m}),(\bullet, 112.0 \mu \mathrm{m})$ and $(+, 216.5 \mu \mathrm{m})$. The symbols and radii for droplets with thickness spacing of $\Delta=13.1 \mu \mathrm{m}$ are $(\square, 50.0 \mu \mathrm{m}),(\boldsymbol{\square}, 98.0 \mu \mathrm{m})$ and $(\odot, 177.0 \mu \mathrm{m})$. 

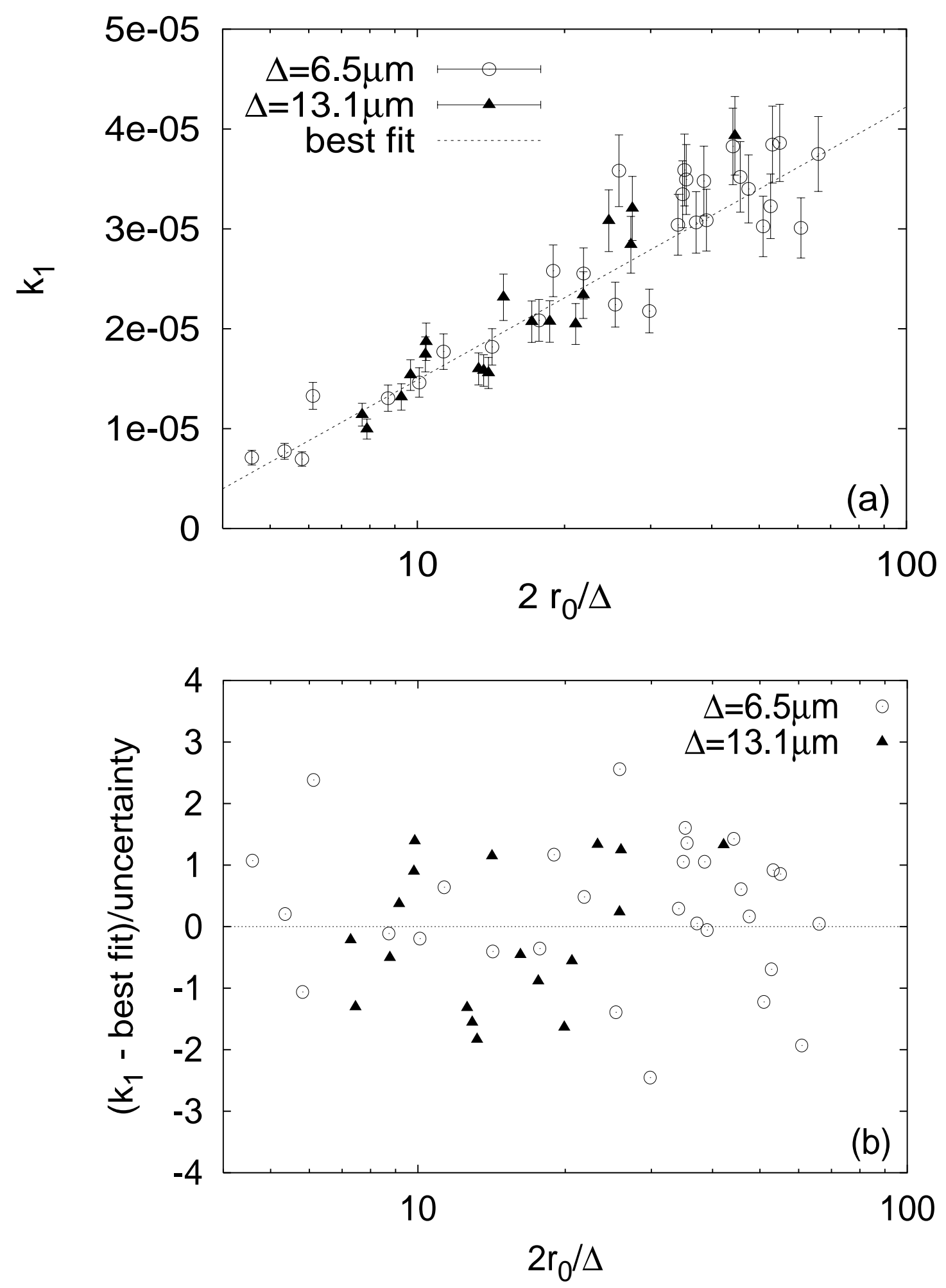

FIG. 5. (a) The plot of $k_{1}$ vs $1 / p=\left(2 r_{0} / \Delta\right)$ on a log scale. The dashed line is the best fit of the data to a straight line. (b) The deviation plot of the data from the best fit normalized by uncertainty of each data point. 

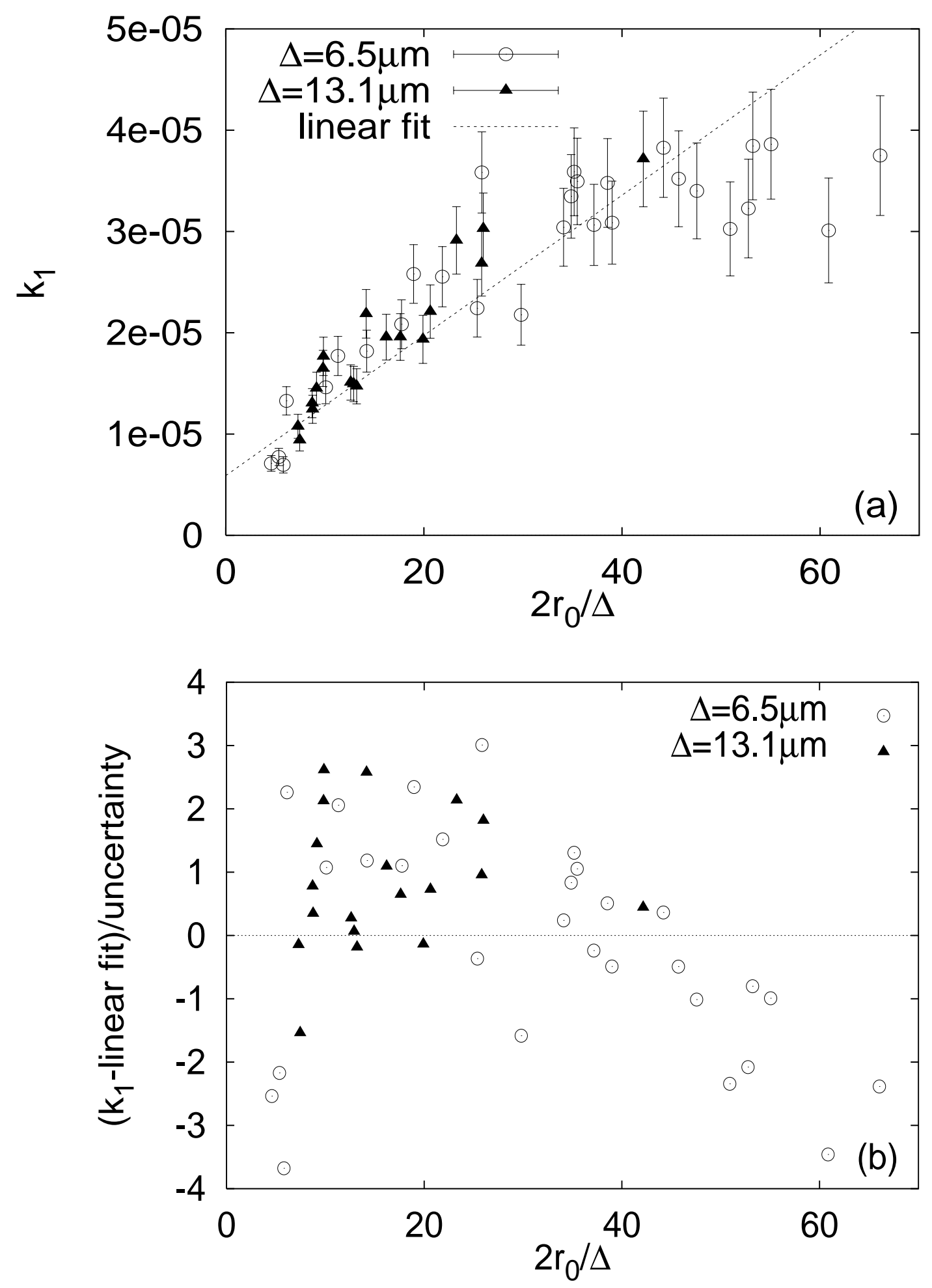

FIG. 6. (a) The plot of $k_{1}$ vs $1 / p=2 r_{0} / \Delta$ and the best fit straight line. (b) The deviation plot of the data from the best linear fit. 


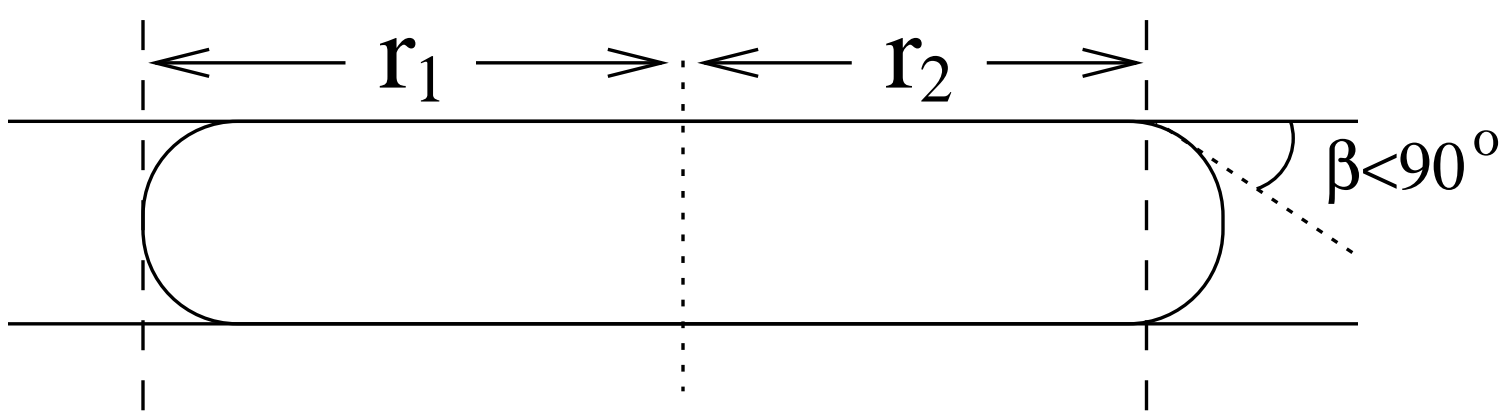

FIG. 7. A ferrofluid droplet making an acute contact angle with the glass plates. 